better than early neurologic examination in predicting outcome. Score ranges of infants with HIE most likely to benefit from hypothermia were identified, but scores were not considered high enough to exclude hypothermia and not suitable for clinical practice. If validated, the models could prove useful in future clinical trials of hypothermia. The authors found them suitable for risk stratification or assessment of prognosis but not as a basis for withdrawal of support.

Cognitive impairment without cerebral palsy as sequel to perinatal asphyxia. Survivors of overt neonatal encephalopathy are at risk of developing cognitive deficits, even in the absence of functional motor deficits, according to a review of the literature (Gonzalez FF, Miller SP. Arch Dis Childhood (Fetal and Neonatal Edition) Nov 2006;91:F454-F459). The cognitive deficits occur in survivors of moderate and severe neonatal encephalopathy, and especially in those with a watershed pattern of injury, involving cortex and white matter in posterior intervascular boundary regions. Advanced MR brain imaging is used to correlate the location and severity of lesions with neurodevelopmental outcome. Whereas cerebral watershed lesions are associated with cognitive impairment without motor deficits, basal ganglia-thalamus injury correlates with severely impaired motor and cognitive outcomes. Cognitive deficits include delays in reading, spelling and arithmetic, and an increased risk of attention deficit hyperactivity disorder.

EEG artifacts, derived from electrical or movement interference, influence the voltage and width of the amplitude-integrated EEG (aEEG) band, when used as a continuous recording at the bedside in the NICU (Hagmann CF et al. Pediatrics Dec 2006;118:2552-2554). aEEG is used as a screening tool for selection of infants with neonatal encephalopathy for trials of mild hypothermia. Prediction of neurodevelopmental outcome is possible at 3 to 6 hours after birth and up to 3 days after admission. (Shah DK et al. Pediatrics 2006;118:47-55; Ped Neur Briefs Aug 2006;20:58). The artifacts may lead to erroneous classification of the aEEG trace.

Caffeine may protect prematures from perinatal white matter injury, according to studies in mice reared in hypoxia from postnatal days 3 through 12 (Back SA et al. Ann Neurol Oct 2006;Published Online). Myelination was enhanced and ventriculomegaly reduced in caffeine treated pups. Caffeine is a nonselective adenosine antagonist used to stimulate respiration in premature infants.

\title{
ABNORMAL ORBITOFRONTAL GYRI DUE TO PREMATURITY
}

The depth and volume of the primary olfactory sulcus and secondary orbital sulci in a sample of 22 adolescents with history of very-preterm birth (VPTB), compared to control subjects born at term, were measured, using MRI Anatomist/Brain VISA 3.0.1 package, and possible reductions in gray and white matter analyzed, using voxel-based morphometry (VBM), in a study at University of Barcelona, Spain. Significant reduction was observed in the depth of the secondary orbitofrontal sulci (which begin to develop at 28 weeks'gestation) but not in the primary olfactory sulci (developed at 16 weeks' gestation) of prematurely born compared to term born subjects at adolescence. Orbital sulcal depth reductions were accompanied by reduced gray-matter volume, using VBM analysis. (Gimenez M, Junque C, 
Vendrell $\mathrm{P}$ et al. Abnormal orbitofrontal development due to prematurity. Neurology Nov (2 of 2) 2006;67:1818-1822). (Reprints: Dr Carme Junque, Department of Psychiatry, University of Barcelona, C/ Casanova, 143, 08036 Barcelona, Spain).

COMMENT. The impaired development of the secondary orbital sulci is consistent with the premature birth before 32 weeks, and before these sulci have formed. In contrast, the primary sulci appear at 16 weeks' gestation and are well formed at 25 weeks. The sulcal abnormality appears to correlate with gray-matter volume reduction. The authors conclude that the orbitofrontal sulcal abnormality is a sequel of premature birth or neonatal complications. Special MR techniques are proving useful in detecting subtle structural cerebral defects that might explain cognitive and behavioral developmental deficits.

\section{LEARNING AND LANGUAGE DISORDERS}

\section{MRI ANATOMICAL CORRELATES OF READING AND LANGUAGE DEFICITS}

Neuroanatomical correlates of developmental dyslexia (DD), defined by isolated reading deficits, and specific language impairment (SLI), defined by poor receptive and expressive language skills, were examined using MR imaging in a heterogeneous sample of 14 boys and 8 girls (11-16 yers of age) with learning disabilities, in a study at University of Florida; Georgetown University, Washington, DC; and other centers. Using a quantitative anatomical risk index, children with smaller and symmetrical brain structures (negative risk indices) had severe comprehension impairments typical of SLI, whereas those with larger, asymmetrical brain structures (positive risk indices) had DD (reading deficit with preserved comprehension). Children with normal anatomy and near zero risk indices had the best learning performance. Speed of rapid automatic naming was not related to the anatomical risk index but was predicted by variation in frontal lobe and cerebellar anatomic measures. Comprehension deficits distinguish SLI from DD. (Leonard C, Eckert M, Given B, Virginia $\mathrm{B}$, Eden $\mathrm{G}$. Individual differences in anatomy predict reading and oral language impairments in children. Brain Dec 2006;129:3329-3342). (Respond: Christiana Leonard, Department of Neuroscience, University of Florida, PO Box 100244, Gainesville, FL 32605).

COMMENT. Children with negative anatomical risk indices (smaller symmetrical brain structures including Heschl's gyri) have severe deficits in reading and language function, including comprehension, typical of SLI, whereas children with positive anatomical risk indices (larger cerebral volume and Heschl's gyri, more asymmetrical brain structures, including planum temporale and cerebellar anterior lobe) have fewer learning deficits, with relative sparing of receptive language and reading comprehension, typical of DD. Further research with larger samples is needed to confirm the correlation of the anatomical risk index with reading and language deficits, and the value of MRI studies in predicting learning impairments and need for academic accommodations. 